\title{
Gametocytes of Toxoplasma gondii in tissue culture
}

\author{
Editorial Magda E Azab
}

Medical Parasitology Department, Faculty of Medicine, Ain Shams University, Cairo, Egypt

Toxoplasma gondii, included in the Phylum Apicomplexa, is a tissue parasite in the intermediate host and an intestinal parasite in the feline definitive host and needs no vector. In the tissues, it replicates asexually by endodyogeny, and endopolygeny; and asexually and sexually by schizogony and gametogony in cat ileum giving rise to infective oocysts. In vitro culture in cell lines provide a suitable model for studying proliferation of the parasite. Retrograde examination of permanent preparations demonstrating morphological forms of multiplying Toxoplasma in an in vitro culture cell line, revealed what may be considered gametogonic stages.

Keywords: gametogony, macrogametocyte, microgametocyte, tissue culture, T. gondii.

Received: 30 October, 2020, Accepted: 24 November, 2020.

Corresponding Author: Magda E Azab, Tel. +20 12222108667, E-mail: drmagda_azab@med.asu.edu.eg

Print ISSN: 1687-7942, Online ISSN: 2090-2646, Vol. 13, No. 3, December, 2020.

The decisive breakthrough in the history of T. gondii is the full demonstration of its asexual and sexual life cycle stages in the feline intermediate host, and its recognition as a coccidian, simultaneously in 1970 by Hutchison $^{[1]}$ and Frenkel ${ }^{[2]}$. Before that decisive report, research had aimed at the description of asexual multiplying tachyzoites by endodyogeny into two daughtercells ${ }^{[3,4]}$,forminglimited numbers of merozoites in pseudocysts. Piekarski et $a l^{[5]}$ also described the asexual multiplication into thousands of bradyzoites by endopolygeny in which there is repeated nuclear divisions, and is followed by the formation of walled cysts in the tissues of the vertebrate intermediate hosts. Both zoites proved to be morphologically identical with minor structural modifications ${ }^{[6]}$, despite differences in their infectivity to non-feline vertebrates. Description of the enteric sexual cycle in the feline host showed that bradyzoites released from ingested tissue cysts invade enteroepithelial cells of the ileum and undergo several repeated cycles of asexual endopolygeny divisions in parasitophorous vacuoles ${ }^{[1,2]}$. After several repeated cycles of divisions, released meronts start the sexual cycle by differentiating into enteroepithelial macro- and microgamonts. The microgamont, syn. microgametocyte, develops 16-30 microgametes ${ }^{[7]}$. In microgametogenesis divided nuclei move to the periphery covered by a budding of its cytoplasm together with a portion of the mitochondrium and two basal bodies ${ }^{[6]}$. The mature microgametes detach from the residual cytoplasm bodies and appear as elongated spiral structures with an electron dense nucleus anterior to which is the mitochondrion and two basal bodies from which originate two flagella. In the Apicomplexa, life cycle stages of the parasites lack protruding flagella except for the male gametes that possess two typical 9 doublet microtubules and a central pair $(9+2)$ axonemes attached to basal body structures ${ }^{[8]}$. Microgametes use their flagella to swim and penetrate and fertilize mature macrogametes forming zygotes. After fertilization, an oocyst wall is formed around the parasite. Infected epithelial cells rupture and discharge oocysts into the intestinal lumen to mix with the stools and contaminate the environment ${ }^{[9]}$.

Because Toxoplasma is an obligate intracellular parasite that multiplies in any nucleated cell, tissue culture cell lines provide a suitable model for studying proliferation of the parasite. One such study ${ }^{[10]}$ cultured the virulent RH strain of Toxoplasma in a WI-38 cell line originally prepared from human embryonic lung, in Eagle's Minimum Essential Meduim supplemented with foetal calf serum and penicillin/streptomycin $(100$ units $/ \mathrm{ml}$ per $100 \mu \mathrm{g} / \mathrm{ml}$ ), and maintained at $37^{\circ} \mathrm{C}$ in $5 \%$ $\mathrm{CO}_{2}$. This report captured images of in vitro multiplying forms in parasitophorous vacuoles in appropriately fixed slide preparations stained with haematoxylin and eosin, and Giemsa. Developmental sequence of schizogonic forms was described and proved to belong to the RH strain by fluorescent antibody staining using specific antisera. In that report ${ }^{[10]}$, the described developmental sequence of schizogonic forms refutes the preposition that these could be precystic stages. Generally, multiplying toxoplasms by endodyogeny, endopolygeny or schizogony are encompassed in parasitophorous vacuoles, while tissue cysts have thick walls and are not surrounded by vacuolar space. In the year 2016, Francia et al. ${ }^{[8]}$ declared the difficulty of growing microgametes in vitro, limiting their use in molecular applications.

Recently in the year 2020, re-examination of the same slide preparation of schizogony forms from the work reported by Azab and Beverely ${ }^{[10]}$, I came across another smaller acidophilic nucleated cytoplasmic form surrounded by a parasitophorous vacuole in preparations from $4^{\text {th }}, 5^{\text {th }}$, and $7^{\text {th }}$ days of culture (Fig. 1). This was probably an early microgametocyte (Mc) precursor of another form seen in the same vicinity. This second form (Mg) apparently composed 


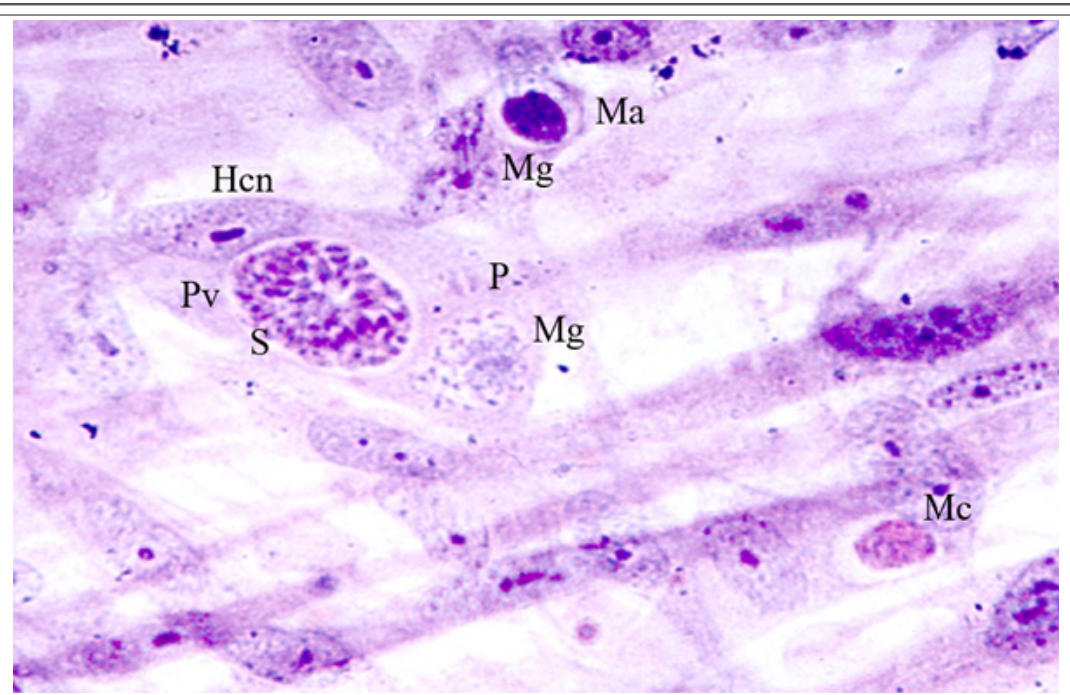

Fig. 1. Light micrograph of RH strain in WI-38 cell line (H\&E).

Multiple infections in the same host cell shows a differentiated schizont (S) of many merozoites and a residual mass of cytoplasm within a parasitophorous vacuole (Pv), adjacent to the host cell nucleus (Hcn). Within the cytoplasm of the same host cell, is another form that contains small coma-shaped microgamete-like organisms with lightly stained nuclei (Mg), surrounding a residual mass of cytoplasm, and enclosed in a parasitophorous vacuole. The difference in shape and size of the organisms in the two forms is quite evident. The cytoplasm of the same host cell contains a pair of toxoplasms (P); evidence of endodyogeny. An acidophilic multi nucleated cytoplasm mass in a parasitophorous vacuole (Mc), and adjacent to the host cell nucleus is probably the microgametocyte precursor of $(\mathrm{Mg})$. Another strongly basophilic form also in a parasitophorous vacuole and adjacent to the host cell nucleus is a macrogametocyte-like form (Ma) (x1000).

of a number of coma-shaped nucleated bodies and a residual mass of cytoplasm, altogether surrounded by a parasitophorous vacuole, represents developed microgametes. These nucleated small and faintly basophilic forms resembled very much preparations of microgametes described by Hutchison et al. ${ }^{[1]}$ in smears made from the intestinal epithelium of the cat. Unfortunately, number, and demonstration of detailed morphology of flagellated microgametes was not possible at this magnification (x1000). In the same described photomicrograph (Fig.1), an intra-cellular basophilic oval macrogametocyte-like form (Ma), also in a parasitophorous vacuole, was seen in the vicinity of the microgametes. This I believe is the only record of gametogenesis of Toxoplasma grown in in vitro cultured cells. Herein, registering the possibility of growing macro- and microgametocytes in vitro is a breakthrough for advanced research at the structural and molecular level for studying genetic variation of $T$. gondii. Research on gene expression data or proteomes from distinct stages of gametogenesis, is required for understanding the biogenesis pathways.

\section{Concluding remarks}

1. At that time (1970), our study ${ }^{[10]}$ of in vitro cultured forms of Toxoplasma coincided with the attempts of demonstrating enteroepithelial schizogony and gametogony, later published in 1971 by Hutchison et al. ${ }^{[11]}$.

2. Regrettably, we missed the important confirmation that these are indeed gametogonic forms. The supernatant of the culture dishes should have been examined for released oocysts.

\section{REFERENCES}

1. Hutchison WM, Dunachie JF, Siim JC, Work K. Coccidianlike nature of Toxoplasma gondii. Brit Med J 1970; 1: 142 144.

2. Frenkel J K, Dubey JP, Miller NL. Toxoplasma gondii in cats: Fecal stages identified as cocciclian oocysts. Science 1970; 167: 893-8963-

3. Goldman M, Carver RK, Sulzer AJ. Reproduction of Toxoplasma gondii by internal budding. J Parasit 1958; 44: 161-171

4. van der Zypen E, Piekarski G. Endodyogeny in Toxoplasma gondii. A morphological analysis. Z. Parasitenkd 1967; 9:1535 (In German.)

5. Piekarski G, Pelster B, Witte H M. Endopolygeny in Toxoplasma gondii. Z Parasitenkd 1971; 36:122-130

6. Dubey JP, Lindsay DS, Speer CA. Structures of Toxoplasma gondii tachyzoites, bradyzoites, and sporozoites and biology and development of tissue cysts.. Clin Microbiol Rev 1998; 11(2): 267-299.

7. Ferguson DJP. Toxoplasma gondii: 1908-2008, homage to Nicolle, Manceaux and Splendore. Mem Inst Oswaldo Cruz, Rio de Janeiro 2009; 104(2): 133-148

8. Francia MA, Dubremetz JF, Morrissette NS. Basal body structure and composition in the apicomplexans Toxoplasma and Plasmodium. Cilia 2016; 5: 3.

9. Dubey JP, Frenkel JK. Cyst-induced toxoplasmosis in cats. J Protozool 1972;19: 155-177.

10. Azab M, Beverley JK. Schizogony of Toxoplasma gondii in tissue cultures. Z Parasitenkd 1974; 44(1):33-41.

11. Hutchison WM, Dunachie JF, Work K, Siim JC. The life cycle of the coccidian parasite, Toxoplasma gondii, in the domestic cat. Trans Roy Soc Trop Med Hyg 1971; 65(3), 380-399. 